\title{
ABSTRACT
}

\section{Structure determination by microcrystal electron diffraction}

\section{Brent L. Nannenga}

brent.nannenga@asu.edu

A common barrier to high-resolution structure determination is the growth of large wellordered crystals. Electron diffraction is capable of producing high-quality diffraction data from crystals that are orders of magnitude smaller than those needed for conventional X-ray crystallographic experiments, and the method of microcrystal electron diffraction (MicroED) has begun to yield high-resolution structures from extremely small microcrystals. In this presentation, the cryo-electron microscopy technique of MicroED will be described in detail along with representative structures determined by the method. Additionally, current work in our lab, which is focused on improving MicroED methodology and extending this technique to new samples will be presented. 